\title{
Children With Attention Deficits Concentrate Better After Walk in the Park
}

\author{
Andrea Faber Taylor \\ Frances E. Kuo \\ University of Illinois, Urbana-Champaign
}

\begin{abstract}
Objective: In the general population, attention is reliably enhanced after exposure to certain physical environments, particularly natural environments. This study examined the impacts of environments on attention in children with ADHD. Method: In this within subjects design, each participant experienced each of three treatments (environments) in single blind controlled trials. Seventeen children 7 to 12 years old professionally diagnosed with ADHD experienced each of three environments - a city park and two other well-kept urban settings - via individually guided 20-minute walks. Environments were experienced 1 week apart, with randomized assignment to treatment order. After each walk, concentration was measured using Digit Span Backwards. Results: Children with ADHD concentrated better after the walk in the park than after the downtown walk $(\mathrm{p}=.0229)$ or the neighborhood walk $(\mathrm{p}=.0072)$. Effect sizes were substantial (Cohen's $\mathrm{d}=.52$ and .77 , respectively) and comparable to those reported for recent formulations of methylphenidate. Conclusion: Twenty minutes in a park setting was sufficient to elevate attention performance relative to the same amount of time in other settings. These findings indicate that environments can enhance attention not only in the general population but also in ADHD populations. "Doses of nature" might serve as a safe, inexpensive, widely accessible new tool in the tool kit for managing ADHD symptoms. (J. of Att. Dis. 2008; XX(X) 1-XX)
\end{abstract}

Keywords: children; attention; physical environment; symptom management

A central puzzle about ADHD is that although the deficits are chronic and generally severe, they are not consistent (Rosenthal, Riccio, Gsanger, \& Jarratt, 2006; Shue \& Douglas, 1992). In children with attention deficits, while performance on tasks involving attention is generally substantially below same-age peers, it is also occasionally good and sometimes excellent (Barkley, 1995). As Barkley (1995) has observed, "the problem...is not that they cannot do the work" but that they cannot maintain this level of performance the way most children can (p. 41). The fluctuations in symptoms are so striking that clinicians have argued that ADHD is better described not as an attention deficit but as an attention inconsistency (Hallowell \& Ratey, 1994).

When and why do ADHD symptoms temporarily disappear? Currently, the short-term fluctuations in attention deficit symptoms are neither well characterized nor well understood. A handful of studies have begun to identify systematic patterns in the fluctuations (Dane, Schachar, \& Tannock, 2000; Porrino et al., 1983; Steenari et al., 2003; Urschitz et al., 2004; Zagar, 1983); overall, however, the study of variability in ADHD symptoms is in its infancy. A more precise characterization of when and why the deficits abate could yield insights into ADHD's etiology and treatment; it might also help address the challenge these short-term fluctuations pose for accurate diagnosis (DuPaul \& Barkley, 1992).

This work brings theory and evidence originally developed in another field to bear on ADHD and its puzzling fluctuations in symptomatology. Attention Restoration Theory (ART) was originally developed in environmental psychology to explain why individuals in

Authors' Note: Many thanks to Johanna Weber for her contributions to literature and manuscript review, and data collection, and to Janet Zoch, Christina Carr, Hamsi Yedavalli, Jessica Carrillo, Pilar Rodriguez, and Jessica Gremp for their contributions to data collection. This material is based on work supported by the National Urban and Community Forestry Advisory Council, U.S. Forest Service under award No. 00DG-11244225-354, and the Cooperative State Research, Education and Extension Service, U.S. Department of Agriculture, under Project No. ILLU-65-0370. Please address correspondence to Andrea Faber Taylor, Landscape and Human Health Laboratory, University of Illinois, Urbana - Champaign, 1101 W. Peabody Drive, Urbana, IL 61801, afabrtay@illinois.edu. 
the general population consistently reported a sense of rejuvenation after wilderness outings and other exposures to relatively natural environments. A body of evidence now indicates that nonclinical samples of both adults and children perform systematically better on objective measures of attention after spending time in or viewing natural surroundings (Faber Taylor, Kuo, \& Sullivan, 2002; Kuo, 2001; Tennessen \& Cimprich, 1995).

Three tenets of ART offer potential insights into the nature of ADHD. First, ART, which is based on work by William James, posits that attention draws on two different mechanisms: one for deliberately directed, effortful forms of attention, and another for involuntary, effortless forms of attention (James, 1962; Kaplan, 1995). The notion of two mechanisms underlying attention may partially explain why individuals with ADHD can routinely sustain focus on tasks they find interesting (i.e., tasks drawing primarily on involuntary attention) but are unable to do so for tasks they find uninteresting (i.e., tasks drawing primarily on effortful, directed attention; Antrop, Roeyers, Van Oost, \& Buysse 2000; Zentall, 1993). An ART-based interpretation of ADHD would be that the disorder involves the mechanism underlying effortful attention, leaving the mechanism underlying involuntary forms of attention intact.

Second, ART posits that the mechanism underlying deliberately directed attention is subject to fatigue and restoration (Kaplan, 1995). With overuse, the theory goes, this form of attention becomes increasingly effortful and ineffective. With rest - that is, during sleep and during gently absorbing activities drawing primarily on involuntary attention - the directed attention mechanism recovers (Kaplan, 1995). The notion of directed attention fatigue and recovery may help explain why ADHD individuals' performance generally worsens over the course of the day (Zagar, 1983; Porrino et al., 1983; Dane et al., 2000 ), and varies as a function of sleep quality and quantity (Steenari et al., 2003; Urschitz et al., 2004).

Third, ART posits that different environments have different effects on attention. ART holds that environments that draw on more effortful forms of attention are fatiguing; that is, extended exposure to such environments results in increasingly poor, increasingly effortful performance on tasks requiring the deliberate use of attention (Kaplan, 1995). By contrast, environments that are gently absorbing and thus draw predominantly on involuntary attention can be restorative; exposure to such environments can allow the mechanism underlying directed attention to rest and rejuvenate (Kaplan, 1995). To the extent that children with ADHD are subject to attention fatigue and recovery, then ART would predict that different environments should have different effects on attention in these children; in particular, natural environments should improve attention in children with ADHD.

There's some evidence to indicate this may be true. Two recent studies of children with ADHD suggest that attention deficit symptoms may decrease after activities in more natural or greener outdoor settings. In surveys on how after-school activities affect children's ADHD symptoms, parent ratings consistently indicate that symptoms show more improvement after activities in relatively natural settings than after activities in indoor or relatively built outdoor settings (Faber Taylor et al., 2001; Kuo \& Faber Taylor, 2004). In both a national study involving more than 450 participants and a more in-depth study involving 96 participants, improvements in attention have been reported across a variety of subpopulations and settings (Faber Taylor et al., 2001; Kuo \& Faber Taylor, 2004). This study seeks to determine whether these reported improvements are real by using controlled exposures to different settings and measuring attention objectively.

\section{Methods}

This study examined effects of exposure to different physical environments on attention in children with ADHD. Single blind controlled trials were conducted in a within subjects crossover design, with each child serving as their own control.

\section{Participant Characteristics and Recruitment}

Children 7 to 12 years old and professionally diagnosed with ADHD by a physician, psychologist, or psychiatrist were recruited through newspaper advertisements and flyers for a study on "how children's after-school activities can affect the severity of ADHD symptoms." Advertisements were placed in local media outlets-print newspapers and an electronic newsletter distributed to university students and staff. Flyers were posted in local health care centers and local public and private grade schools.

Twenty-five children entered the program. Of those, eight were excluded from further analysis: four experienced errors in procedure, two did not complete the study, one was noncompliant during all three sessions, and one had an outlying Digit Span Backward score (a score 3.5 standard deviation from the child's other scores). The final sample included 17 children, 15 boys, and 2 girls. The ratio of boys to girls diagnosed with attention deficits in the general population is 4:1 (American Psychiatric Association, 1994); the gender ratio in this study was not significantly different from that ratio $(p=.31)$. The participants' mean age was 9.23 years. 


\section{Design: Settings and Randomization}

Three settings were examined. Each setting was chosen to represent a common category of public outdoor environments: an urban park, a downtown area, and a residential area. Each setting was well maintained and had roughly equivalent terrain (flat), ambient noise levels (quiet), and levels of pedestrian traffic (minimal).

Each child was exposed to each of the three settings in turn, over the course of three separate sessions. To control for effects of children's increasing familiarity with staff and procedures over time, order of exposures was counterbalanced, with children randomly assigned to experience the settings in one of three orders: $\mathrm{P}(\mathrm{ark})$, D(owntown), N(eighborhood); D, N, P; or N, P, D. With a sample of 17 participants, the best possible counterbalancing of the sequence of conditions (PDN, NPD, or DNP) would have been $6,6,5$. The number of children in each sequence of conditions in the final sample was 6 PDN, 7 NPD, 4 DNP.

Exposure to the different settings was provided via carefully controlled, individual, guided walks. A given child experienced each of the three settings at the same time of day and day of week. All walks were conducted prior to the child receiving any medication for the day. All walks were conducted at the same pace in relatively warm weather, between 8:30 a.m. and 5:30 p.m. during June and early July. The same guide accompanied a given child on all their walks, and conversation during walks was kept to a minimum. To minimize differences in rapport between child and walking guide across sessions, guides spent some time getting acquainted with each of their assigned children before the start of the first session. Thus, this study controlled for time of day and day of week, medication status, level of physical exertion, and social setting across environments, as well as terrain, noise, and social density.

\section{Procedure}

Prior to each of the walks, children completed a series of puzzles. These puzzles were selected to ensure some degree of attentional fatigue, as the positive aftereffects of certain physical environments on attention are thought to reflect recovery from attentional fatigue. Children completed the puzzles in a quiet, indoor facility, accompanied by their assigned walking guide. The child and their walking guide were then driven to their assigned setting for the day. All three settings were within a 2.5to 3-minute drive from the indoor facility.

At the day's assigned setting, children and their guides walked a route designed to be completed in 20 minutes at a relaxed pace. The guides kept the walk on schedule and discouraged conversation.
After the walk, the child and their guide were driven back to the quiet, indoor facility. During the drive, children answered some simple questions about their experience of the walk. Upon arrival at the indoor facility, guides escorted children to a testing room, where the child completed tests of concentration and impulse control. A single test administrator, blind to the children's walking conditions, administered all tests. To enhance children's engagement in the tests, children were shown a treasure chest and told that they could choose a small toy (less than a $\$ 1$ in value) from it after completing the tests.

\section{Measures}

Digit Span Backwards (DSB) is a widely used, standardized measure of concentration. It is sensitive to deficits in attention and executive functioning, and researchers have recommended that it be used in diagnosing ADHD (Hale, Hoeppner, \& Fiorello, 2002). The DSB involves listening to a sequence of numbers from two to eight digits long (e.g., 2-5-1) and repeating the sequence aloud in reverse order (e.g., 1-5-2) (Wechsler, 1955). After a correct response, a new, longer sequence is given; after an incorrect response, respondents are given another sequence of the same length to attempt. Scores on DSB reflect the number of digits in the longest sequence a participant successfully reversed before two consecutive failed trials (Wechsler, 1955).

Three other measures were administered after DSB: the Stroop Color-Word Test (Dodrill, 1978), Symbol Digit Modalities (SDM) (Smith, 1968), and the Vigilance Task of the Gordon Diagnostic System Model 111-R (VT) (Gordon, McClure, \& Aylward, 1996). Although all four measures have been widely used to test attention and impulse control in ADHD and other populations in between subjects designs, Stroop displayed practice effects (demonstrated in a repeated measures ANOVA comparing scores by session number) $F(2,17)=16.90$, $p<.0001$, and Stroop, SDM, and VT displayed low power (power $=.05, .28$, and.07, respectively, in repeated measures ANOVAs comparing scores by condition). Thus, these measures are not further described here. In retrospect, the lack of power in a repeated measures design with an ADHD population is not surprising, as one of the hallmarks of ADHD is high variability in performance, particularly on multi-trial tasks (Barkley, 1990).

To assess children's experience of the different walks, each child was asked whether they found their walk fun, relaxing, interesting, scary, boring, weird, and/or uncomfortable. Children responded to each description on a 3 -point scale (not at all $=0$, kinda $=1$; and very $=2$ ). Not sure was also an option. 
To gather general information about the children, we asked parents to complete a short survey. The survey included questions about diagnoses and medication patterns.

\section{Results}

Of the 16 children whose parents completed the parent survey, 6 were diagnosed with ADD and 10 diagnosed with ADHD. Forty-four percent of the children were diagnosed with at least one comorbid disorder; $56 \%$ had no comorbidities. While none of the children were taking medication on the days they participated in the study, $50 \%$ used medication daily year round; $19 \%$ used medication on a variable schedule such as "weekdays only" or "only during the school year," and $31 \%$ did not use medications at all to treat their ADHD symptoms.

Digit Span Backwards, a measure of concentration, demonstrated adequate power (power $=.75$ ) in a repeated measures ANOVA comparing scores by condition, and demonstrated no effects of practice, $F(2,16)=1.37, p=.27$, in a repeated measures ANOVA comparing scores by session number.

Analysis of the Digit Span Backwards scores showed that children's ability to concentrate varied significantly as a function of their previous surroundings. A repeated measures ANOVA indicated that DSB performance varied by setting, $F(2,16)=4.72, p=.02$ (see Figure 1).

On closer examination, this effect reflects the difference between the park setting versus the other two, less natural settings. The two less natural settings showed no significant differences in concentration; DSB scores after the neighborhood and downtown settings were not significantly different from each other $(M=3.71$ and 3.82 , respectively; $S D=1.21$ and $1.07 ; p=.64$ ). By contrast, concentration after the park exposure was significantly better than after either of the other two settings. The DSB scores after the park walk were significantly better than after the neighborhood walk, Fisher's protected least significant difference (Fisher's PLSD) $d=$ $.71, p=.007$, and significantly better than after the downtown walk, Fisher's PLSD $d=.59, p=.02$.

The effect of the park exposure on DSB performance was substantial. Expressed in terms of digit span, DSB performance was roughly six-tenths of a digit better after the park exposure than after the downtown exposure $(d=$ $.59, M=4.41$ vs. $M=3.82, S D=1.18$ and $S D=1.07$ ), and roughly seven-tenths of a digit better after the park exposure than after the neighborhood exposure $(d=.71$, $M=4.41$ versus $M=3.71, S D=1.18$ and $S D=1.21$ ). By way of comparison, Table 1 summarizes findings from

\section{Figure 1 \\ Mean Postwalk Scores on Digit Span Backwards for Park, Neighborhood, and Downtown Conditions}

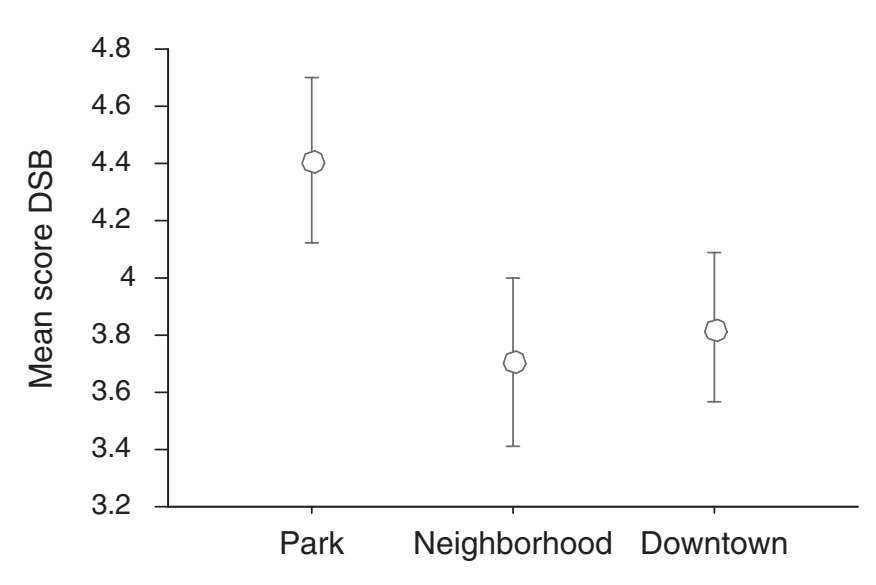

studies reporting DSB performance in ADHD samples and same age peers. As Table 1 shows, ADHD groups most commonly score roughly six-tenths of a digit worse than groups without ADHD. Thus, the effect of the park walk observed here is roughly equal and opposite to the performance deficit due to ADHD.

We also compared the effect sizes observed here with recent effect sizes reported for methylphenidate on a cognitive performance measure roughly comparable to DSB. Expressed in terms of Cohen's $d$, the advantage of the park walk over the downtown and neighborhood exposures was .52 and .77 , respectively. By way of comparison, Cohen's $d$ for the effects of two extendedrelease formulations of methylphenidate were .59 and .54 , for Metadate $\mathrm{CD}$ (methylphenidate $\mathrm{HCl}$, US Pharmacopeia) and Concerta (methylphenidate $\mathrm{HCl}$ ), respectively (Swanson, Wigal, \& Wigal, 2004). Those $d$ s reflect the peak effects of these two extended-release formulations. Thus, the effect of the park walk observed here is roughly equal to the peak effects of two typical ADHD medications.

One-tailed $t$ tests were used to compare the children's ratings of the walk settings. Attention restoration theory (Kaplan, 1995) would predict that settings yielding better concentration would also be subjectively experienced as more restorative. Thus, we expected that the children's experiential ratings of the different settings would follow the same pattern as their DSB scores. Indeed, children's ratings of the three settings mirrored their concentration performance for the three settings. The downtown and neighborhood did not differ significantly in children's ratings of their experience. By contrast, the park setting was experienced significantly more positively than the other 
Table 1

Mean Scores and Differences in Mean Scores on Digit Span Backwards Reported in Studies Comparing Peer Groups With and Without ADHD

\begin{tabular}{|c|c|c|c|}
\hline Reference & $\begin{array}{l}\text { Control Group } \\
\text { mean score DSB }\end{array}$ & $\begin{array}{l}\text { ADHD Group } \\
\text { mean score DSB }\end{array}$ & $\begin{array}{l}\text { Difference in } \\
\text { DSB mean scores }\end{array}$ \\
\hline $\begin{array}{l}\text { Willcutt E. G., Pennington B. F., \& Olson, R. K., et al.. } \\
\text { (2005). Dev Neuropsychol }\end{array}$ & 6.0 & 4.9 & 1.1 \\
\hline $\begin{array}{l}\text { Rosenthal E. N., Riccio, C. A, Gsanger, K. M., et al. } \\
\text { (2006). Arch Clin Neuropsychol. }\end{array}$ & 4.22 & $\begin{array}{l}3.5 \\
\text { (combined type) }\end{array}$ & $\begin{array}{c}.72 \\
\text { (combined type) }\end{array}$ \\
\hline Wu, K. K., Anderson, V., \& Castiello, U. (2002). Dev Neuropsychol. & 5.24 & 4.56 & .68 \\
\hline Karatekin, C., \& Asarnow, R. F. (1998). Psychiatry Res. & 4.9 & 4.3 & .6 \\
\hline Oie, M., Sundet, K., \& Rund, B. R. (1999). Neuropsychologia & 4.48 & 3.89 & .59 \\
\hline $\begin{array}{l}\text { Barkley, R. A., Edwards, G., Laneri, M., et al. } \\
\text { (2001). J Abnorm Child Psychol }\end{array}$ & 6.9 & 6.4 & .50 \\
\hline Rucklidge, J. J., \& Tannock, R. (2002). J Child Psychol Psychiatry & 7.23 & 6.73 & .50 \\
\hline $\begin{array}{l}\text { Rosenthal, E. N., Riccio, C. A., Gsanger, K. M., et al. } \\
\text { (2006). Arch Clin Neuropsychol. }\end{array}$ & 4.22 & $\begin{array}{c}4.67 \\
\text { (predominantly } \\
\text { inattentive) }\end{array}$ & $\begin{array}{c}-.45 \\
\text { (predominantly } \\
\text { inattentive) }\end{array}$ \\
\hline Shue, K. L., \& Douglas, V. I. (1992). Brain Cogn. & 3.83 & 4.18 & -.35 \\
\hline
\end{tabular}

Note: DSB $=$ Digit Span Backwards.

two settings; the park setting was rated significantly higher than those two settings on fun, $t(13)=2.39, p=.02$, one-tailed, and marginally significantly higher on relaxing, $t(14)=1.42, p=.09$, one-tailed, although not on interesting $(p=.83)$. There were no significant differences across settings in children's rating of negative experiences (weird, $p=.43$; boring, $p=.83$; uncomfortable, $p=.43$; or scary, $p=.73$ ).

\section{Discussion}

In this study, we examined the impacts of three settings on children with ADHD. Children with attention deficits concentrated better after walking in a park than after either of two other settings. The effect of a dose of green was substantial-roughly as large as the deficit due to ADHD and roughly as large as the peak effect of extended-release methylphenidate. Children's experience of the three settings showed the same pattern as their attention performance afterward. The park was experienced significantly more positively than the other two settings, and there were no significant differences between the other two settings.

\section{Relation to Previous Theory and Research}

These findings are consistent with ART. Attention Restoration Theory would predict that to the extent that children with attention deficits are susceptible to attentional fatigue, exposing them to a relatively natural environment might enhance their attention performance afterward.
Furthermore, ART would predict that (other things being equal) whichever settings children found most gently absorbing in this study would also have the best effects on performance. According to the theory, gently absorbing settings draw on involuntary attention, providing a respite from more effortful, deliberately directed modes of attention. Although we did not ask children how "gently absorbing" each of the settings were, the children rated the park setting as both more relaxing and more fun than the other two settings, with the other two settings being rated not significantly different. Mirroring this pattern, children's attention performance was best after the park setting and not significantly different after the other two settings.

These findings reinforce and extend previous research. In the last couple decades, considerable research in the general population has indicated that exposure to natural settings yields reliable, positive aftereffects on attention (for review, see Kuo, 2001). Two studies have explored the possibility that exposure to relatively natural settings might relieve ADHD symptoms. Although both studies were based on parent report rather than objective measures and were based on unsystematic comparisons across settings, the findings were highly promising. In surveys on how after-school activities affect their child's ADHD symptoms, parents consistently reported that symptoms improve after activities in relatively natural settings (Faber Taylor et al., 2001; Kuo \& Faber Taylor, 2004) and children who typically played in "greener" outdoor settings displayed milder symptoms overall than children who typically played in less green settings (Faber 
Taylor et al., 2001). Further, data from over 450 parents nationwide suggested the link between improved symptoms and activities done in greener settings held for boys and girls ages 5-18 years old, living in different geographic regions and different sized communities, from rural areas to the inner city (Kuo \& Faber Taylor, 2004). From the survey responses, it would appear that the restorative benefits of nature hold for a wide variety of activities ranging from reading, to games, to sports (Faber Taylor et al., 2001; Kuo \& Faber Taylor, 2004).

The strengths and weaknesses of the present study complement those of the two previous studies involving ADHD populations. The use of carefully controlled comparisons across settings and an objective performance measure of attention in this study helps to address critical limitations in the previous, survey-based work. At the same time, the strengths of the previous work - the use of a national sample and a wide range of participants engaged in a wide range of activities in a wide range of settings-help address the limited sampling in the present study. Thus, the effects of doses of nature on attention are not limited to the particular activity (i.e., walking in pairs) or the particular setting (i.e., an urban park) examined in this study. Taken together, the findings from this study and the previous large-scale survey studies suggest that not only does exposure to nature enhance attention in children with ADHD, but also that this effect holds for a wide variety of children, settings, and activities.

\section{Implications for the Understanding and Treatment of ADHD}

These findings point to a potential explanation for a central puzzle about ADHD: If attention performance is intact some of the time, why is it so often so compromised? Previous findings suggest that different physical environments have different effects on attention in the general population; the findings here suggest that the same is true of children with ADHD. Thus, the marked fluctuations in performance typical of ADHD may reflect differential exposure to attentionally fatiguing and restorative environments, and more generally, attention fatigue and recovery brought on by different tasks and activities. It would be interesting to further explore the relationship between attention deficits and attention fatigue. It seems likely that ADHD might confer a particular susceptibility to fatigue; an even more intriguing possibility is that the disorder is, in essence, a severe susceptibility to attention fatigue.

As we evaluate doses of nature as a potential treatment for ADHD, several questions remain. First, what is the time course of effects? Certainly the peak effects found here are substantial, even after a brief 20-minute exposure, and previous, survey-based findings suggest that the effects last at least long enough for parents to have noticed them. But the current study examined the effects on attention only immediately after environmental exposure; at present, we have no objective performance data showing that the effects of nature doses last long enough to be of practical use in managing ADHD symptoms.

A second, important question is whether the benefits of nature extend not only to attention but also to the other major difficulty in ADHD: impulse control. In the general population, there is evidence that both attention and impulse control are enhanced by exposure to nature (Faber Taylor et al., 2002); to the extent that the effects of nature exposure on attention generalize to ADHD populations, it would seem likely for the effects on impulse control to generalize as well. Future research should examine whether nature doses reduce both inattention and impulsivity in ADHD.

A third question is whether doses of nature help with actual academic performance. Attention is a crucial factor in academic performance (Duncan et al, 2007), and the specific measure used in this study, DSB, predicts academic performance (Jackson \& Myers, 1982), so it would not be surprising to find that doses of nature improve academic performance. At the same time, direct evidence as to whether doses of nature improve academic performance would be helpful in assessing its value as a potential treatment.

We suggest that the potential for using doses of nature to help treat ADHD deserves prompt attention. The current tool kit of treatments is not effective for all sufferers (Barkley, 2006; Rowland, Lesesne, \& Abramowitz, 2002) and in many cases offers only limited relief from symptoms (MTA Cooperative Group, 2004; National Institute of Health, 1998). Behavioral therapies are typically not sufficient to bring children into normal ranges of functioning (Antshel \& Remer, 2003; Fiore, Becker, \& Nero, 1993; Hinshaw, 1994), and medications entail some risks and side effects (MTA Cooperative Group, 2004; National Institute of Health, 1998; Rowland et al., 2002).

As a possible, additional tool in the tool kit for treating ADHD, doses of nature have several attractive qualities. Spending time in relatively natural outdoor areas does not entail any unusual risks or negative side effects, nor is there any social stigma associated with spending time outdoors. The kinds of settings examined in the previous, survey-based research are widely available and generally cost nothing. To the extent that spending time outside is enjoyable (recall that our participants found the park walk most relaxing and most fun) and that the benefits of nature exposure appear to hold across a wide 
variety of activities, adherence to a treatment regime including doses of nature might be quite high. Furthermore, although this study indicates that a park setting is best, previous findings suggest that a park-like setting is not necessary for beneficial effects and that frequent visits to a particular natural setting may not diminish the effects of that setting (Faber Taylor et al., 2001). Finally, directing children to spend more time outdoors might have some positive side effects. Recently, a study examining more than 7,000 children found that in urban areas, availability of residential green space is linked to a lower incidence of obesity, controlling for individual sociodemographics and neighborhood socioeconomic status (Liu, Wilson, Qi, \& Ying, 2007). Encouraging children to spend time outdoors might not only reduce ADHD symptoms but also increase physical activity and its benefits.

\section{Possible Implications for Schools}

It is interesting to consider how doses of nature might be used in school settings, given that attention is an important resource in learning (Duncan et al., 2007). If doses of nature prove to be a viable treatment in the school setting, there are many ways schools might administer such a treatment. Schools might consider including more natural elements and areas in schoolyards-trees, ornamental shrubs, open lawn, and flower or vegetable gardens, which in turn attract wildlife. Access to the natural areas and the outdoors might be provided by way of recess, physical education classes, and environmentally based lessons or curricula (e.g., writing, science or math lessons could focus on natural elements). Natural spaces created indoors might also provide restoration from symptoms (Katcher \& Wilkins, 2000), particularly if they provide the characteristics theorized as necessary for attention restoration to occur (Kaplan, 1995; Kaplan, Kaplan, \& Ryan, 1998). A quiet space, separated from the hustle and bustle of daily activities, and with aquariums, terrariums, and indoor plants might be effective. Finally, window views of natural settings have been shown to foster greater attentional functioning in the general population (Faber Taylor et al., 2002; Kaplan, 2001; Tennessen \& Cimprich, 1995); green views from the classroom might help children with and without attention deficits stay on task and learn more in the course of the school day. Consistent with the possibility that natural environments might enhance student performance, a study of 40 schools and more than 400 students found that environmentally based curricula were linked with a whole range of benefits, including increased engagement and enthusiasm for learning and higher scores on standardized tests in reading, writing, math, science, and social studies (Lieberman \& Hoody, 1998).

\section{References}

American Psychiatric Association. (1994). Diagnostic and statistical manual of mental disorders (4th ed.). Washington, DC: American Psychiatric Press.

Antrop, I., Roeyers, H., Van Oost, P., \& Buysse, A. (2000). Stimulation seeking and hyperactivity in children with ADHD. Journal of Child Psychology \& Psychiatry, 41(2), 225-231.

Antshel, K., \& Remer, R. (2003). Social skills training in children with attention deficit hyperactivity disorder: A randomizedcontrolled clinical trial. Journal of Clinical Child \& Adolescent Psychology, 32, 153-165.

Barkley, R. A. (1990). Attention Deficit Hyperactivity Disorder: A handbook for diagnosis and treatment. New York: Guilford Press.

Barkley, R. A. (1995). Taking charge of ADHD. New York: Guilford Press.

Barkley, R. A. (2006). Attention-Deficit Hyperactivity Disorder. A handbook for diagnosis and treatment (3rd ed.). New York: Guilford Press.

Dane, A. V., Schachar, R. J., \& Tannock, R. (2000). Does actigraphy differentiate ADHD subtypes in a clinical research setting? Journal of the American Academy of Child and Adolescent Psychiatry, 39(6), 752-760.

Dodrill, C. B. (1978). A neuropsychological battery for epilepsy. Epilepsia, 19, 611-623.

DuPaul, G. J., \& Barkley, R. A. (1992). Situational variability of attention problems - psychometric properties of the revised home and school situations questionnaires. Journal of Clinical Child Psychology, 21, 178-188.

Duncan, G. J., Claessens A., Huston A. C., Pagani, L. S., Engel M., Sexton H., et al. (2007). School readiness and later achievement. Developmental Psychology, 43(6), 1428-1446.

Faber Taylor, A., Kuo, F. E., \& Sullivan, W. (2001). Coping with ADD: The surprising connection to green play settings. Environment and Behavior, 33, 54-77.

Faber Taylor, A., Kuo, F. E., \& Sullivan, W. C. (2002). Views of nature and self-discipline: Evidence from inner city children. Journal of Environmental Psychology, 22, 49-64.

Fiore, T. A., Becker, E. A., \& Nero, R. C. (1993). Interventions for students with attention deficits. Exceptional Children: Special Issue: Issues in Education of Children with Attention Deficit Disorder, 60(2), 163-173.

Gordon, M., McClure, D. F., \& Aylward, G. P. (1996). The Gordon Diagnostic System: Interpretive guide. DeWitt, NY: GSI Publications.

Hale, J. B., Hoeppner, J. B., \& Fiorello, C. A. (2002). Analyzing digit span components for assessment of attention processes. Journal of Psychoeducational Assessment, 20, 128-143.

Hallowell, E., \& Ratey, J. (1994). Driven to distraction: Recognizing and coping with attention deficit disorder from childhood through adulthood. New York: Pantheon.

Hinshaw, S. P. (1994). Attention deficits and hyperactivity in children (Vol. 29). Thousand Oaks, CA: Sage.

Jackson, N. E., \& Myers, M. G. (1982). Letter naming time, digit span, and precocious reading-achievement. Intelligence, 6, 311-329.

James, W. (1962). Psychology: The briefer course. New York: Collier Books. (Original work published 1892).

Kaplan, R. (2001). The nature of the view from home: Psychological benefits. Environment \& Behavior, 33, 507-542.

Kaplan, S. (1995). The restorative benefits of nature: Toward an integrative framework. Journal of Environmental Psychology, 15, 169-182. 
Kaplan, R., Kaplan, S., \& Ryan, R. (1998) With people in mind: Design and management of everyday nature. Washington, DC: Island Press.

Katcher, A. H., \& Wilkins, G. G. (2000). The centaur's lessons: Therapeutic education through care of animals and nature study. In A. Fine (Ed.), Handbook on animal-assisted therapy: Theoretical foundations and guidelines for practice (pp. 153-177). San Diego: Academic Press.

Kuo, F. (2001). Coping with poverty: Impacts of environment and attention in the inner city. Environment and Behavior, 33, 5-34.

Kuo, F. E., \& Faber Taylor, A. (2004). A potential natural treatment for Attention-Deficit/Hyperactivity Disorder: Evidence from a national study. American Journal of Public Health, 94, 1580-1586.

Lieberman, G. A., \& Hoody, L. (1998). Closing the achievement gap. San Diego, CA: State Education and Environment Roundtable.

Liu, G. C., Wilson, J. S., Qi, R., \& Ying, J., (2007). Green neighborhoods, food retail and childhood overweight: Differences by population density. American Journal of Health Promotion, 21(4), 317-325.

MTA Cooperative Group. (2004). National Institute of Mental Health Multimodal Treatment Study of ADHD follow-up: 24-month outcomes of treatment strategies for attention-deficit/hyperactivity disorder. Pediatrics, 113, 754-761.

National Institute of Health. (1998). Diagnosis and treatment of Attention-Deficit/Hyperactivity Disorder (ADHD). NIH Consensus Statement, 16, 1-45.

Porrino, L. J., Rapoport, J. L., Behar, D., Sceery, W., Ismond, D. R., \& Bunney, W. E. (1983). A naturalistic assessment of the motor activity of hyperactive boys. Archives General Psychiatry, 40, 681-687.

Rosenthal, E. N., Riccio, C. A., Gsanger, K. M., \& Jarratt, K. P. (2006). Digit Span components as predictors of attention problems and executive functioning in children. Archives of Clinical Neuropsychology, 21, 131-139.

Rowland, A. S., Lesesne, C. A., \& Abramowitz, A. J. (2002). The epidemiology of Attention-Deficit/Hyperactivity Disorder (ADHD): A public health view. Mental Retardation \& Developmental Disabilities Research Reviews, 8, 162-170.
Shue, K. L., \& Douglas, V. I. (1992). Attention-deficit hyperactivity disorder and the frontal-lobe syndrome. Brain and Cognition, 20, 104-124.

Smith, A. (1968). The Symbol Digit Modalities Test: A neuropsychologic test for economic screening of learning and other cerebral disorders. Learning Disorders, 3, 83-91.

Steenari, M. R., Vuontela, V., Paavonen, E. J., Carlson, S., Fjallberg, M., \& Aronen, E. T. (2003) Working memory and sleep in 6- to 13-year-old schoolchildren. Journal of the American Academy of Child and Adolescent Psychiatry, 42(1), 85-92.

Swanson, J. M., Wigal, S. B., Wigal, T., Sonuga-Barke, E., Greenhill, L. L., Biederman, J., et al. (2004). A comparison of once-daily extended-release methylphenidate formulations in children with Attention-Deficit/Hyperactivity Disorder in the laboratory school (the Comacs study). Pediatrics, 113, e206-e216.

Tennessen, C. M., \& Cimprich, B. (1995). Views to nature: Effects on attention. Journal of Environmental Psychology, 15, 77-85.

Urschitz, M. S., Eitner, S., Guenther, A., Eggebrecht, E., Wolff, J., Urschitz-Duprat, P. M., et al. (2004). Habitual snoring, intermittent hypoxia, and impaired behavior in primary school children. Pediatrics, 114(4), 1041-1048.

Wechsler, D. (1955).Wechsler Adult Intelligence Scale manual. New York: Psychological Corporation.

Zagar, R., \& Bowers, N. D. (1983). The effect of time of day on problem solving and classroom behavior. Psychology in the Schools, 20, 337-345.

Zentall, S. S. (1993). Research on the educational implications of attention deficit hyperactivity disorder. Exceptional Children, 60, 143-152.

Andrea Faber Taylor is a postdoctoral research scientist studying children's environments and behavior in the Department of Natural Resources and Environmental Sciences at the University of Illinois, Urbana-Champaign.

Frances E. Kuo is an associate professor in the Department of Natural Resources and Environmental Sciences, and the Department of Psychology at University of Illinois, UrbanaChampaign. 